\title{
Prognostic Significance of Diastolic Dysfunction With Multiple Comorbidities in Heart Failure Patients
}

\author{
Baldeep Mann ${ }^{1}$, Janpreet S. Bhandohal ${ }^{2}$, Savi Mushiyev ${ }^{3}$ \\ 1. Internal Medicine, Metropolitan Hospital Center, New York Medical College, New York, USA 2. Internal Medicine, \\ University of California, Los Angeles - Kern Medical Center, Bakersfield, USA 3. Cardiology, Metropolitan Hospital \\ Center, New York Medical College, New York, USA
}

Corresponding author: Janpreet S. Bhandohal, jpsbhandohal87@gmail.com

\section{Abstract}

\section{Background}

Heart failure poses a significant burden on health care and economy. In recent years, diastolic dysfunction has been increasingly recognized as a significant predictor of readmission in heart failure patients.

\section{Objectives}

We aimed to identify factors predicting readmission in patients with clinical heart failure at 30 days and six months.

\section{Methods}

A retrospective chart review was performed at a single urban medical center, including 208 patients in our final analysis.

\section{Results}

A higher Charlson comorbidity index (CCI) and moderate anemia (hemoglobin $[\mathrm{Hb}]<10 \mathrm{~g} / \mathrm{dL}$ ) were significant predictors of readmission at both 30 days and six months. In addition, advanced chronic kidney disease (CKD) stage (4 or 5) and follow-up in a cardiology clinic were significant predictors at six months. During multivariate analysis, worsening diastolic dysfunction (grade 3 or 4) (OR: 2.09; 95\% CI: 1.03 to 4.23), higher CCI (OR: 1.18; 95\% CI: 1.03-1.36), and Hb < 10 g/dL (OR: 3.42; 95\% CI: 1.44-8.13) were independent predictors of readmission at 30 days. Higher CCI (OR: 1.37; 95\% CI: 1.19-1.58) and CKD stage 4 or 5 (OR: 3.05; 95\% CI: 1.40-6.62) were independent predictors of readmission at six months.

Received 05/03/2020 Review began 05/08/2020 Review ended 05/16/2020 Published 05/26/2020

() Copyright 2020 Mann et al. This is an open access article distributed under the terms of the Creative Commons Attribution License CC-BY 4.0., which permits unrestricted use, distribution, and reproduction in any medium, provided the original author and source are credited.

\section{Conclusions}

Worse diastolic dysfunction (grade 3 or 4) was a significant predictor of all-cause readmission at 30 days post-discharge in heart failure patients. Higher CCI precisely predicted readmission as an independent variable at 30 days and six months. Anemia $(\mathrm{Hb}<10 \mathrm{~g} / \mathrm{dL})$ and CKD stage 4 or 5 were significant predictors of readmission at 30-days and six months, respectively.

Categories: Cardiology, Endocrinology/Diabetes/Metabolism, Infectious Disease

Keywords: diastolic dysfunction, heart failure, readmission

\section{Introduction}

Heart failure (HF) affects an estimated 6.5 million Americans $\geqslant 20$ years of age and has an expected increase in prevalence by $46 \%$ from 2012 to 2030 [1]. It is one of the leading causes of morbidity and mortality and poses a high economic burden on both the patient and the society due to high health-care-related costs projecting from $\$ 24.7$ billion in 2010 to $\$ 77.7$ billion in 2030 in the United States [2,3]. The incidence of HF rises sharply with advancing age, especially over 60 years [1,2]. Previously, HF was most often categorized into HF with reduced ejection fraction (HFrEF) and HF with preserved ejection fraction (HFpEF), but, currently, three categories are used based on left ventricular ejection fraction (LVEF): $\operatorname{HFrEF}$ (LVEF $<40 \%$ ), HFpEF (LVEF $\geqslant 50 \%$ ), and LVEF of $40 \%-49 \%$ categorized as mid-range (HFmrEF) [4]. Approximately $50 \%$ of patients with the clinical syndrome of $\mathrm{HF}$ have a preserved ejection fraction (HFpEF) in the community. Multimorbidity is common in HF but appears to be more severe in HFpEF as compared with HFrEF [5]. It has therefore become increasingly important to recognize and address diastolic dysfunction and to also determine the role of multiple comorbidities in the prognosis of patients with HF.

\section{Materials And Methods}


We aimed to study the relationship between grades of diastolic dysfunction and rehospitalization rate, as an independent factor and in combination with comorbidities, among all HF patients (including HFrEF and HFpEF) admitted to the Metropolitan Hospital Center (MHC), New York. We conducted a single-center retrospective chart review of patients aged 18 years or older with HF admitted to the center between July 1 , 2010, and June 30, 2016. Patient charts were reviewed using electronic medical records. Data of patients with HFrEF (LVEF < 40\%) and HFpEF (LVEF > 40\%) were reviewed. First admission in MHC with symptoms or signs of HF was considered as index admission. Follow-up records were reviewed till six months from index admission to determine the number of readmissions at 30 days and six months. If a patient was admitted within 30 days, six months were calculated from the subsequent admission to eliminate duplication. Inclusion criteria were patients aged 18 years and above admitted with symptoms and signs of HF such as shortness of breath (New York Heart Association class I-IV), orthopnea and paroxysmal nocturnal dyspnea, pedal edema, elevated jugular venous distention, crepitation on lung auscultation, including both HFrEF and HFpEF, patients with baseline echocardiography to determine ejection fraction (using modified Simpson's method) as well as the degree of diastolic dysfunction during index admission in this hospital, and patients with HF who had other labs and investigations performed such as NT-pro BNP (NT-proB-type natriuretic peptide), BUN (blood urea nitrogen), serum creatinine, hemoglobin ( $\mathrm{Hb}$ ) A1c. Serum creatinine was used to determine chronic kidney disease (CKD) staging in patients with underlying renal dysfunction [6]. Exclusion criteria include patients with HF who expired or left the hospital without echocardiography or assessment of cardiac function, patients who lost to follow-up post-hospital discharge (also including the patients who were admitted to other hospitals based on history), and patients with any terminal illness with life expectancy less than six months. In addition, patients were excluded if they had arrhythmias, paced rhythm, or poor quality of echocardiography, which would impair the determination of diastolic dysfunction. Patients who were admitted mainly for hemodialysis or ambulatory surgery were also excluded.

The primary aim of this study was to determine whether diastolic dysfunction plays a role in all-cause readmission in patients with $\mathrm{HF}$. The secondary aim was to determine the burden of other comorbidities in predicting readmission. Charlson comorbidity index (CCI) was used as a criterion to determine the comorbidity burden. The study was performed in compliance with the BRANY IRB and STAR NYC-HHC protocol. Statistical analysis was performed using SPSS Version 25.0 (IBM Corp., Armonk, NY, USA). Chisquare test was used for categorical variables, and Student's t-test was used for continuous variables. Multivariate analysis was performed using logistic regressions. A p-value of $<0.05$ was considered statistically significant.

\section{Results}

A total of 208 patients were finally included in the analysis (Figure 1). The various characteristics and factors determining readmission at 30 days and six months are given in Tables 1 and 2, respectively. Majority of our patients were males (119 [57.2\%]). The mean age in the readmission group was higher than the nonreadmission group at both 30 days and six months, so was the comorbidity burden as determined by CCI. CCI was reported as a continuous variable. Other variables based on laboratory results were categorized in various ways. Lower $\mathrm{Hb}$ was analyzed having the presence or absence of moderate anemia $(\mathrm{Hb}<10 \mathrm{~g} / \mathrm{dL})$, troponin I was categorized as either positive or negative ( $>0.04$ as being positive), and CKD staging was analyzed by comparing outcomes of stage $4 / 5$ as compared to other stages (1/2/3). LVEF was categorized as either reduced or preserved (LVEF $<40 \%$ was considered reduced). The diastolic dysfunction was categorized into grades 0 to 4 based on echocardiographic criteria and was analyzed comparing grade 3 or 4 with grade 02 [7]. Other echocardiographic variables that were taken into account included left atrial volume index (LAVI), left ventricular end-diastolic diameter (LVEDD), left atrial diameter (LAD), and right ventricular systolic pressure (RVSP), all of them reported as continuous variables. Length of stay was another continuous variable. Patients with appropriate follow-up in the cardiology clinic (seven days post-discharge) were reported as categorical variables. 


\section{Cureus}

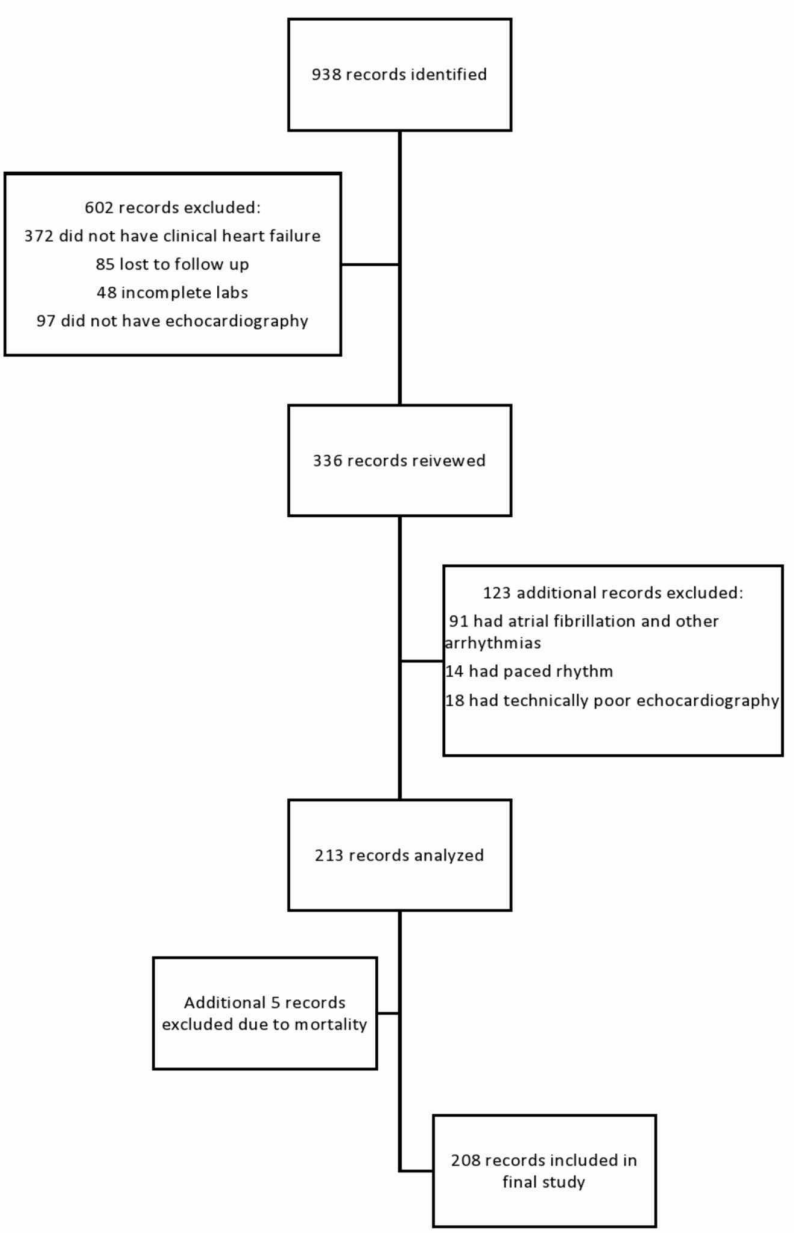

FIGURE 1: Flowchart showing the selection process of cases. 


\section{Cureus}

\begin{tabular}{|c|c|c|c|}
\hline Characteristics (30-day readmission) & Yes & No & p-Value \\
\hline Total & $53(25.48 \%)$ & $155(74.52 \%)$ & \\
\hline Male gender & $26(49.1 \%)$ & $93(60.0 \%)$ & 0.11 \\
\hline Age & 65.28 (SD: 11.98) & 63.32 (SD: 13.28) & 0.342 \\
\hline HTN not controlled & $19(35.8 \%)$ & $72(46.5 \%)$ & 0.118 \\
\hline Charlson comorbidity index & 5.66 (SD: 2.26) & 4.74 (SD: 2.29) & 0.012 \\
\hline $\mathrm{Hb}<10 \mathrm{~g} / \mathrm{dL}$ & 17 (32.1\%) & $19(12.3 \%)$ & 0.002 \\
\hline Troponin I positive (>0.04) & $23(43.4 \%)$ & $77(49.7 \%)$ & 0.264 \\
\hline CK & 169.74 (SD: 149.87) & 210.37 (SD: 198.48) & 0.174 \\
\hline HbA1c & 6.99 (SD: 2.03) & 7.07 (SD: 2.11) & 0.817 \\
\hline CKD stage 4/5 & $15(28.3 \%)$ & $31(20.0 \%)$ & 0.144 \\
\hline DD grade $3 / 4$ & $20(37.7 \%)$ & $42(27.1 \%)$ & 0.10 \\
\hline LVEF $<40 \%$ & $31(58.5 \%)$ & $107(69.0 \%)$ & 0.10 \\
\hline $\operatorname{LVEDD}(\mathrm{cm})$ & 5.54 (SD: 0.95) & 5.58 (SD: 0.81) & 0.757 \\
\hline LAD (cm) & 4.33 (SD: 0.54) & 4.32 (SD: 0.58) & 0.962 \\
\hline RVSP (mm Hg) & 39.00 (SD: 11.66) & 37.26 (SD: 13.13) & 0.393 \\
\hline Length of stay (days) & 4.89 (SD: 4.46) & 4.31 (SD: 4.06) & 0.385 \\
\hline Seen in the cardiology clinic (7 days) & $11(20.8 \%)$ & $50(32.3 \%)$ & 0.077 \\
\hline
\end{tabular}

\section{TABLE 1: Factors predicting 30-day readmission.}

SD, standard deviation; HTN, hypertension; Hb, hemoglobin; CKD, chronic kidney disease; DD, diastolic dysfunction; LVEF, left ventricular ejection fraction; LVEDD, left ventricular end-diastolic diameter; LAD, left atrial diameter; RVSP, right ventricular systolic pressure. 


\section{Cureus}

\begin{tabular}{|c|c|c|c|}
\hline Characteristics (six months readmission) & Yes & No & p-Value \\
\hline Total & 102 (49\%) & $106(51 \%)$ & \\
\hline Male gender & $59(57.8 \%)$ & $60(56.6 \%)$ & 0.484 \\
\hline Age & 65.27(SD: 13.34) & 62.42 (SD: 12.50) & 0.112 \\
\hline HTN not controlled & $43(42.2 \%)$ & $48(45.3 \%)$ & 0.377 \\
\hline Charlson comorbidity index & 5.65 (SD: 2.56) & 4.33 (SD: 1.84) & 0.000 \\
\hline $\mathrm{Hb}<10 \mathrm{~g} / \mathrm{dL}$ & $25(24.5 \%)$ & $11(10.4 \%)$ & 0.006 \\
\hline Troponin I positive (>0.04) & $48(47.1 \%)$ & $52(49.1 \%)$ & 0.441 \\
\hline CK & 190.30 (SD: 156.56) & 209.37 (SD: 213.93) & 0.466 \\
\hline HbA1c & 6.84 (SD: 1.79) & 7.25 (SD: 2.32) & 0.160 \\
\hline CKD stage 4/5 & $33(32.4 \%)$ & $13(12.3 \%)$ & 0.000 \\
\hline DD grade $3 / 4$ & $33(32.4 \%)$ & $29(27.4 \%)$ & 0.263 \\
\hline LVEF $<40 \%$ & $62(60.8 \%)$ & $76(71.7 \%)$ & 0.064 \\
\hline LVEDD (cm) & 5.61 (SD: 0.84) & 5.54 (SD: 0.85) & 0.557 \\
\hline LAD (cm) & 4.34 (SD: 0.52) & 4.30 (SD: 0.62) & 0.638 \\
\hline RVSP (mm Hg) & 38.00 (SD: 12.25) & 37.42 (SD: 13.30) & 0.746 \\
\hline Length of stay (days) & 4.96 (SD: 5.00) & 3.97 (SD: 3.10$)$ & 0.087 \\
\hline Seen in the cardiology clinic (7 days) & $24(23.5 \%)$ & $37(34.9 \%)$ & 0.049 \\
\hline
\end{tabular}

\section{TABLE 2: Factors predicting six-month readmission.}

SD, standard deviation; HTN, hypertension; Hb, hemoglobin; CKD, chronic kidney disease; DD, diastolic dysfunction; LVEF, left ventricular ejection fraction; LVEDD, left ventricular end-diastolic diameter; LAD, left atrial diameter; RVSP, right ventricular systolic pressure.

In both 30-day readmission and six-month readmission data, higher CCI and moderate anemia $(\mathrm{Hb}<10$ $\mathrm{g} / \mathrm{dL}$ ) were found to be significant predictors (Table 1). In addition, advanced CKD staging (stage 4/5) and follow-up in the cardiology clinic at seven days (clinic visit being associated with lower readmission rate) were significant predictors of readmission at six months (Table 2). On multivariate analysis, worsening diastolic dysfunction, higher CCI, and decreased $\mathrm{Hb}$ were independent predictors of readmission at 30 days (Table 3). Higher CCI and worsening renal dysfunction were independent predictors of readmission at six months (Table 4). Diastolic dysfunction was not statistically significant at six months in predicting the readmission independently.

\begin{tabular}{|c|c|c|}
\hline Readmission (30 days) & Logistic regression adjusted OR $[95 \% \mathrm{Cl}]$ & p-Value \\
\hline DD grade $(0-2$ vs. $3 / 4)$ & 2.09 [1.03-4.23] & 0.041 \\
\hline Charlson comorbidity index & $1.18[1.03-1.36]$ & 0.018 \\
\hline CKD stage $4 / 5$ & $1.005[.43-2.32]$ & 0.99 \\
\hline $\mathrm{Hb}<10 \mathrm{~g} / \mathrm{dL}$ & 3.42 [1.44-8.13] & 0.005 \\
\hline
\end{tabular}

\section{TABLE 3: Multivariate analysis for factors predicting 30-day readmission.}

OR, odds ratio; $\mathrm{Cl}$, confidence interval; $\mathrm{DD}$, diastolic dysfunction; CKD, chronic kidney disease; Hb, hemoglobin. 


\section{Cureus}

\begin{tabular}{|c|c|c|}
\hline Readmission (six months) & Logistic regression adjusted OR [95\% Cl] & p-Value \\
\hline DD grade $(0-2$ vs. $3 / 4)$ & $1.43[0.76-2.7]$ & 0.262 \\
\hline Charlson comorbidity index & $1.37[1.19-1.58]$ & 0.001 \\
\hline CKD stage $4 / 5$ & $3.05[1.40-6.62]$ & 0.005 \\
\hline $\mathrm{Hb}<10 \mathrm{~g} / \mathrm{dL}$ & $2.187[0.927-5.163]$ & 0.074 \\
\hline
\end{tabular}

TABLE 4: Multivariate analysis for factors predicting six-month readmission.

OR, odds ratio; $\mathrm{Cl}$, confidence interval; $\mathrm{DD}$, diastolic dysfunction; $\mathrm{CKD}$, chronic kidney disease; Hb, hemoglobin.

LVEF was not a significant predictor for readmission. Uncontrolled hypertension, elevated troponin I, and worse $\mathrm{HbA1c}$ (indicating worse diabetes control) were not associated with readmission. Likewise, none of the echocardiographic parameters such as LVEDD, LAD, and RVSP were useful in predicting readmission. LAVI was not available for all the patients; therefore, a subgroup analysis was performed on 123 patients, which showed that LAVI was not statistically significant in predicting readmission independently at 30 days and six months ( $\mathrm{p}=0.88$ and $\mathrm{p}=0.99$, respectively). Similarly, another subgroup analysis was performed on 178 patients who had ProBNP measured. Logistic regression showed that it was statistically significant in predicting 30-day readmission (OR: 1.0000310; 95\% CI: 1.0000059-1.0000562; $\mathrm{p}=0.015$ ) and six-month readmission (OR: 1.000045 ; 95\% CI: $1.00001-1.00008 ; \mathrm{p}=0.01$ ) but given the extremely small OR would lack clinical significance.

\section{Discussion}

There has been a paucity of data on prognosis in HF patients with diastolic dysfunction as compared with systolic dysfunction. The prevalence of diastolic dysfunction is on the upsurge, especially in the elderly, as compared with systolic dysfunction, which seems to have decreased in the 21st century [2]. In recent years, several studies have been published signifying the role of diastolic dysfunction in various groups of populations. A higher incidence of diastolic dysfunction has been found in patients with chronic obstructive pulmonary disease (COPD) [8]. It has also been associated with mechanical ventilation weaning failure, as characterized by increased E/e' ratio [9]. Diastolic dysfunction is more common in septic patients compared with those with systolic dysfunction and has been associated with increased mortality in this group of patients, with the latter not linked to mortality [10]. Diastolic dysfunction is also associated with death, prolonged mechanical ventilation, and prolonged hospital and ICU length of stay independent of systolic dysfunction in patients undergoing cardiac surgery [11]. Perioperative diastolic dysfunction is also an independent predictor of adverse cardiovascular outcomes in patients undergoing noncardiac surgery [12]. It has also been found to be strongly associated with NAFLD (nonalcoholic fatty liver disease), a hepatic manifestation of metabolic syndrome which is on the rise in the U.S. population [13].

The quality of life, clinical symptoms, readmission rate, and six-month mortality were found to be similar in HF patients with systolic dysfunction and isolated diastolic dysfunction [14]. At the end of one year, left ventricular diastolic dysfunction was also found to be the only echocardiographic predictor of rehospitalization in survivors of acute myocardial infarction [15]. No differences were noted in mortality and readmission rate between HFrEF and HFpEF at the end of the three-year follow-up in the Japanese population [16]. It has therefore become increasingly important to interpret the status of diastolic dysfunction in patients admitted to the hospital. It can no longer be seen as an isolated cardiac dysfunction, rather it has systemic implications. Data from the OPTIMIZE-HF (Organized Program to Initiate Lifesaving Treatment in Hospitalized Patients with Heart Failure) registry revealed similar post-discharge mortality risk and equally high rates of rehospitalization in patients with HFrEF and HFpEF [17]. Restrictive filling pattern is also found to be a powerful predictor of HF hospitalization and mortality in patients postmyocardial infarction $[18,19]$. In another study, combining early trans-mitral flow velocity (E)/early diastolic velocity of mitral annulus (E') with LV ejection fraction was a better predictor of readmission and cardiac death in HF patients compared with LV ejection fraction alone [20].

In our study, we found that worsening diastolic dysfunction was an independent predictor of readmission at 30 days post-discharge but not at six months after index admission for HF during multivariate analysis. These results suggest that patients with worse diastolic dysfunction were either admitted within 30 days or were not admitted even at six months. One possible explanation for this could be that the patients who had better disease control during the initial phase post-discharge were better able to maintain lifestyle modification and medication compliance so that they were not readmitted later regardless of initial diastolic dysfunction grade. On the reverse, non-compliance with medications and follow-up might have played a role for 30-day readmission in patients with worse diastolic dysfunction. Although used to determine diastolic dysfunction wherever available, left atrial volume index was not a significant predictor 
independently. LVEF was also not a significant predictor for readmission. Patients who were not admitted initially, during the six-month follow-up, comorbidities played a major role in readmission, especially renal dysfunction. These findings are similar to what Miyagishima et al. have found [16]. They also concluded that the presence or absence of reduced LVEF was not useful in predicting the prognosis, and, on the other hand, anemia and renal dysfunction were identified as significant prognostic factors [16]. Anemia has been found to be associated with increased long-term mortality rate in patients with diastolic HF [21]. Lower Hb along with other comorbidities such as diabetes, eGFR (estimated glomerular filtration rate), and lower blood pressure were associated with poor outcomes in patients with HFpEF [22]. In this study, Hb of less than 10 $\mathrm{g} / \mathrm{dL}$ was associated with higher readmission at 30 days and six months but was only an independent predictor at 30 days. One might consider severe anemia with/without bleeding as a predisposing factor for early readmission. Due to the extremely small OR for ProBNP, an ROC (receiver operating characteristic) analysis was performed. It suggested a link between the categorical value of ProBNP greater than 3,577 for 30-day readmission and greater than 7,540 for six-month readmission. These cutoff values were statistically significant during regression analysis in predicting readmission (OR: 3.38, 95\% CI: $1.58-7.24, \mathrm{p}=$ 0.001 , specificity $=0.5227$, sensitivity $=0.7556$ for 30 days; and OR: $3.40,95 \%$ CI: $1.73-6.69, p=0.0004$, specificity $=0.8068$, and sensitivity $=0.4494$ for six months). Whether these threshold values have any clinical significance needs to be further evaluated in future studies.

The most significant predictors for readmission in our study at 30 days and six months were higher Charlson comorbidity index and worse renal dysfunction (CKD stage 4 and 5). Similar findings were seen in a study conducted on national database concluded that higher comorbidity burden as determined by CCI in patients of HF with diastolic dysfunction predicted higher readmission rate at 30 days, as was the renal failure [23]. Similar results were found in a large number of COPD patients in whom CCI provided meaningful prediction for readmission at 30 days [24]. Another large-scale study conducted on the Chinese population determined that higher CCI was independently associated with a risk of 30-day readmission in dialysis patients (both hemodialysis and peritoneal dialysis) [25]. CCI has also been found to be an important determinant for length of stay, mortality, and rehospitalization in the elderly [26].

Our study has a few limitations worth mentioning. First, it is a retrospective chart review and a relatively small-sized study conducted at a single center. Our hospital mainly caters to Hispanic and African-American population, which might compromise the generalizability to other patient populations. Secondly, the sample size is relatively small, possibly resulting in statistically non-significant results in certain outcomes such as length of stay, which might otherwise become significant with a bigger sample size. Third, patient follow-up was limited to six months; having a longer follow-up would better determine the long-term implications of worse diastolic dysfunction and comorbidities. Fourth, other variables such as psychosocial issues and insured versus uninsured were not included as part of variables.

\section{Conclusions}

In our study, we concluded that worse diastolic dysfunction (grade 3 or 4) independently predicted all-cause readmission at 30 days post-discharge in HF patients. CCI precisely predicted readmission as an independent variable at 30 days and six months. Anemia $(\mathrm{Hb}<10 \mathrm{~g} / \mathrm{dL})$ and CKD stage 4 or 5 were significant predictors of readmission at 30 days and six months, respectively. LVEF was not a significant predictor of readmission.

\section{Additional Information \\ Disclosures}

Human subjects: All authors have confirmed that this study did not involve human participants or tissue. Animal subjects: All authors have confirmed that this study did not involve animal subjects or tissue. Conflicts of interest: In compliance with the ICMJE uniform disclosure form, all authors declare the following: Payment/services info: All authors have declared that no financial support was received from any organization for the submitted work. Financial relationships: All authors have declared that they have no financial relationships at present or within the previous three years with any organizations that might have an interest in the submitted work. Other relationships: All authors have declared that there are no other relationships or activities that could appear to have influenced the submitted work.

\section{References}

1. Benjamin EJ, Blaha MJ, Chiuve SE, et al.: Heart disease and stroke statistics-2017 update: a report from the American Heart Association. Circulation. 2017, 135:e146-e603. 10.1161/CIR.0000000000000485

2. van Riet EE, Hoes AW, Wagenaar KP, et al.: Epidemiology of heart failure: the prevalence of heart failure and ventricular dysfunction in older adults over time. A systematic review. Eur J Heart Fail. 2016, 18:242252. 10.1002/ejhf.483

3. Tung YC, Chou SH, Liu KL, et al.: Worse prognosis in heart failure patients with 30-day readmission . Acta Cardiol Sin. 2016, 32:698-707. 10.6515/ACS20151113A

4. Bouthoorn S, Valstar GB, Gohar A, et al.: The prevalence of left ventricular diastolic dysfunction and heart failure with preserved ejection fraction in men and women with type 2 diabetes: a systematic review and meta-analysis. Diab Vasc Dis Res. 2018, 15:477-493. 10.1177/1479164118787415

5. Dunlay SM, Roger VL, Redfield MM: Epidemiology of heart failure with preserved ejection fraction . Nat Rev Cardiol. 2017, 14:591-602. 10.1038/nrcardio.2017.65 
6. Levey AS, Eckardt KU, Tsukamoto Y, et al.: Definition and classification of chronic kidney disease: a position statement from Kidney Disease: Improving Global Outcomes (KDIGO). Kidney Int. 2005, 67:2089-2100. 10.1111/j.1523-1755.2005.00365.x

7. Nagueh SF, Smiseth OA, Appleton CP, et al.: Recommendations for the evaluation of left ventricular diastolic function by echocardiography: an update from the American Society of Echocardiography and the European Association of Cardiovascular Imaging. J Am Soc Echocardiogr. 2016, 29:277-314. 10.1016/j.echo.2016.01.011

8. Zhyvotovska A, Yusupov D, Kamran H, et al.: Diastolic dysfunction in patients with chronic obstructive pulmonary disease: a meta-analysis of case controlled studies. Int J Clin Res Trials. 2019, 4:137. 10.15344/2456-8007/2019/137

9. de Meirelles Almeida CA, Nedel WL, Morais VD, Boniatti MM, de Almeida-Filho OC: Diastolic dysfunction as a predictor of weaning failure: a systematic review and meta-analysis. J Crit Care. 2016, 34:135-141. 10.1016/j.jcrc.2016.03.007

10. Sanfilippo F, Corredor C, Fletcher N, Landesberg G, Benedetto U, Foex P, Cecconi M: Diastolic dysfunction and mortality in septic patients: a systematic review and meta-analysis. Intensive Care Med. 2015, 41:10041013. 10.1007/s00134-015-3748-7

11. Metkus TS, Suarez-Pierre A, Crawford TC, et al.: Diastolic dysfunction is common and predicts outcome after cardiac surgery. J Cardiothorac Surg. 2018, 13:67. 10.1186/s13019-018-0744-3

12. Fayad A, Ansari MT, Yang H, et al.: Perioperative diastolic dysfunction in patients undergoing noncardiac surgery is an independent risk factor for cardiovascular events: a systematic review and meta-analysis. Anesthesiology. 2016, 125:72-91.

13. Wijarnpreecha K, Lou S, Panjawatanan P, Cheungpasitporn W, Pungpapong S, Lukens FJ, Ungprasert P: Association between diastolic cardiac dysfunction and nonalcoholic fatty liver disease: a systematic review and meta-analysis. Dig Liver Dis. 2018, 50:1166-1175. 10.1016/j.dld.2018.09.004

14. Badano LP, Albanese MC, De Biaggio P, Rozbowsky P, Miani D, Fresco C, Fioretti PM: Prevalence, clinical characteristics, quality of life, and prognosis of patients with congestive heart failure and isolated left ventricular diastolic dysfunction. J Am Soc Echocardiogr. 2004, 17:253-261. 10.1016/j.echo.2003.11.002

15. Khumri TM, Reid KJ, Kosiborod M, Spertus JA, Main ML: Usefulness of left ventricular diastolic dysfunction as a predictor of one-year rehospitalization in survivors of acute myocardial infarction. Am J Cardiol. 2009, 103:17-21. 10.1016/j.amjcard.2008.08.049

16. Miyagishima K, Hiramitsu S, Kimura $\mathrm{H}$, et al.: Long term prognosis of chronic heart failure. reduced vs preserved left ventricular ejection fraction. Circ J. 2009, 73:92-99. 10.1253/circj.CJ-07-1016

17. Fonarow GC, Stough WG, Abraham WT, et al.: Characteristics, treatments, and outcomes of patients with preserved systolic functionhospitalized for heart failure: a report from the OPTIMIZE-HF Registry. J Am Coll Cardiol. 2007, 50:768-777. 10.1016/j.jacc.2007.04.064

18. omaratne JB, Whalley GA, Gamble GD, Doughty RN: Restrictive filling pattern is a powerful predictor of heart failure events postacute myocardial infarction and in established heart failure: a literature-based meta-analysis. J Card Fail. 2007, 13:346-352. 10.1016/j.cardfail.2007.01.010

19. Whalley GA, Gamble GD, Doughty RN: Restrictive diastolic filling predicts death after acute myocardial infarction: systematic review and meta-analysis of prospective studies. Heart. 2006, 92:1588-1594. 10.1136/hrt.2005.083055

20. Hirata K, Hyodo E, Hozumi T, et al.: Usefulness of a combination of systolic function by left ventricular ejection fraction and diastolic function by e/e' to predict prognosis in patients with heart failure. Am J Cardiol. 2009, 103:1275-1279. 10.1016/j.amjcard.2009.01.024

21. Tehrani F, Phan A, Morrissey R, Chien C, Rafique A, Schwarz ER: The prognostic value of anemia in patients with diastolic heart failure. Tex Heart Inst J. 2009, 36:220-225. https://www.ncbi.nlm.nih.gov/pmc/articles/PMC2696500/

22. Maréchaux S, Six-Carpentier MM, Bouabdallaoui N, et al.: Prognostic importance of comorbidities in heart failure with preserved left ventricular ejection fraction. Heart Vessels. 2011, 26:313-320. 10.1007/s00380010-0057-5

23. Arora S, Lahewala S, Hassan Virk HU, et al.: Etiologies, trends, and predictors of 30-day readmissions in patients with diastolic heart failure. Am J Cardiol. 2017, 120:616-624. 10.1016/j.amjcard.2017.05.028

24. Buhr RG, Jackson NJ, Kominski GF, Dubinett SM, Ong MK, Mangione CM: Comorbidity and thirty-day hospital readmission odds in chronic obstructive pulmonary disease: a comparison of the Charlson and Elixhauser comorbidity indices. BMC Health Serv Res. 2019, 19:701. 10.1186/s12913-019-4549-4

25. Lin Y, Yang C, Chu H, et al.: Association between the Charlson Comorbidity Index and the risk of 30-day unplanned readmission in patients receiving maintenance dialysis. BMC Nephrol. 2019, 20:363. 10.1186/s12882-019-1538-0

26. Bahrmann A, Benner L, Christ M, Bertsch T, Sieber CC, Katus H, Bahrmann P: The Charlson Comorbidity and Barthel Index predict length of hospital stay, mortality, cardiovascular mortality and rehospitalization in unselected older patients admitted to the emergency department. Aging Clin Exp Res. 2019, 31:12331242. 10.1007/s40520-018-1067-X 